\title{
Eruption of the Deciduous Dentition in the Rhesus Monkey
}

\author{
James A. McNamara, Jr., Douglas L. Foster, and Byron D. Rosenstein \\ Center for Human Growth and Development, Department of Anatomy and Reproductive \\ Endocrinology Program, Department of Obstetrics and Gynecology, \\ The University of Michigan, Ann Arbor, Michigan 48109, USA
}

A limited number of investigations have appeared in the literature which have attempted to estimate the age of rhesus monkeys on the basis of dental development. These studies have shown that tooth emergence can be used as a guide for age estimation of normal, healthy rhesus monkeys.

Twenty-one infant rhesus monkeys ( $M a$ caca mulatta) were used in this study. The animals were fed a high protein laboratory diet and were in good health. The emergence of the deciduous dentition was ascertained at weekly intervals for each animal. The criterion employed for tooth emergence was the appearance of any part of the crown of the tooth above the oral mucosa. Tooth emergence was expressed in number of days after birth and in hundredths of a year (one day $=.00274$ year, 3.65 days $=.01$ year). Since the teeth were observed weekly, it was assumed that most eruption occurred halfway between the inspections and thus one half of a week was subtracted from each observed eruption value.

On the average, the first deciduous teeth to erupt were the mandibular central incisors, closely followed by the maxillary central incisors. After the appearance of these teeth, the follow-

Received for publication August 6, 1976.

Accepted for publication November 10, 1976.

This study was supported in part by USPHS Grants DE-43120, HD-08636 and grants from the Ford Foundation. ing sequence of eruption was noted: mandibular lateral incisors, maxillary lateral incisors, maxillary canines, mandibular canines, maxillary first molars, mandibular first molars and maxillary second molars. The greatest variability in this sequence occurred in the relative eruption of the canines and the first molars. In contrast to the above sequence of tooth eruption, the maxillary canine and maxillary first molars erupted at the same time in six animals and the first molar erupted prior to the canine in two other animals. With respect to the mandible, the first molars and the canines erupted simultaneously in two animals; the first molars erupted before the canines in three other animals.

The average values for the emergence of the deciduous dentition found in the present study are similar to those reported previously by Hurme and Van Wagenen but they are slightly lower than those reported by Schultz. This difference may be related to the criterion employed for tooth exuption (i.e., full emergence of the crown of the tooth) in the latter study. The range of variability reported in the present study is also slightly greater than that found by Hurme and Van Wagenen. The values reported for the age or eruption of the deciduous dentition in the present study, however, agree with previous findings to the extent that the composite of such values can be used to estimate reliably the age of growing $\boldsymbol{M}$ mulatta when birth records are unavailable.

TABLE

Maxillary Decibuous Dentition

\begin{tabular}{lrrrrr|llllll}
\hline Days & $\mathbf{I}_{1}$ & $\mathbf{I}_{2}$ & $\mathbf{C}$ & $\mathbf{M}_{1}$ & $\mathbf{M}_{2}$ & Years & $\mathbf{I}_{1}$ & $\mathbf{I}_{2}$ & $\mathbf{C}$ & $\mathbf{M}_{1}$ & $\mathbf{M}_{2}$ \\
\hline Mean & 26 & 44 & 65 & 75 & 158 & Mean & .08 & .12 & .18 & .21 & .43 \\
S.D. & 15 & 14 & 21 & 17 & 20 & S.D. & .04 & .04 & .06 & .05 & .06 \\
Min. & 7 & 24 & 37 & 45 & 127 & Min. & .02 & .07 & .10 & .12 & .35 \\
Mean & 64 & 92 & 132 & 125 & 193 & Max. & .18 & .25 & .36 & .34 & .53 \\
\hline
\end{tabular}

Mandibular Deciduous Dentition

\begin{tabular}{lrrrrr|llllll}
\hline Days & $\mathbf{I}_{1}$ & $\mathbf{I}_{2}$ & $\mathbf{C}$ & $\mathbf{M}_{\mathbf{1}}$ & $\mathbf{M}_{2}$ & Years & $\mathbf{I}_{1}$ & $\mathbf{I}_{2}$ & $\mathbf{C}$ & $\mathbf{M}_{\mathbf{1}}$ & $\mathbf{M}_{2}$ \\
\hline Mean & $\mathbf{2 4}$ & 35 & 68 & 77 & 140 & Mean & .07 & .10 & .19 & .21 & .38 \\
S.D. & 15 & 19 & 24 & 18 & 23 & S.D. & .04 & .05 & .07 & .05 & .06 \\
Min. & 8 & 16 & 39 & 45 & 105 & Min. & .02 & .04 & .11 & .12 & .29 \\
Max. & 64 & 92 & 142 & 125 & $\mathbf{1 8 8}$ & Max. & .18 & .25 & .39 & .34 & .52 \\
\hline
\end{tabular}

